\title{
Lieux communs cinétiques.
}

De nymphas, nymphettes et sylves

\section{Filippo Fimiani}

\section{OpenEdition}

\section{Journals}

Édition électronique

URL : http://journals.openedition.org/imagesrevues/2962

DOI : 10.4000/imagesrevues.2962

ISSN : $1778-3801$

Éditeur :

Centre d'Histoire et Théorie des Arts, Groupe d'Anthropologie Historique de l'Occident Médiéval, Laboratoire d'Anthropologie Sociale, UMR 8210 Anthropologie et Histoire des Mondes Antiques

\section{Référence électronique}

Filippo Fimiani, « Lieux communs cinétiques. », Images Re-vues [En ligne], Hors-série 4 | 2013, mis en ligne le 18 février 2013, consulté le 31 janvier 2021. URL : http://journals.openedition.org/ imagesrevues/2962 ; DOI : https://doi.org/10.4000/imagesrevues.2962

Ce document a été généré automatiquement le 31 janvier 2021.

\section{(c) (7) (8)}

Images Re-vues est mise à disposition selon les termes de la Licence Creative Commons Attribution Pas d'Utilisation Commerciale 4.0 International. 


\section{Lieux communs cinétiques.}

De nymphas, nymphettes et sylves

\section{Filippo Fimiani}

We are all victims of the dance We are all victims of the dance We met at a dance Was it my dream or yours? Who knows It was so long ago

\section{Exemplifier, modes d'emploi}


1 Des hommes, des femmes, des jeunes filles en fleur et des garçons harassés en pantalons courts, des enfants joyeux, des animaux domestiques: voilà les spectateurs oisifs et décontractés du dimanche qui vont assister en souriant et en bon esprit, avec les immanquables appareils photo et caméras, cigarettes et apéros, au petit spectacle mis en place dans un parc d'un quartier résidentiel de New York City. Il s'agit d'une Maypole school dance, d'une dance pour la venue du Printemps du 1963. Et tout est là, dans deux minutes et quelques secondes d'un épisode - le deuxième de la troisième année, Love among the ruins, du 2007 (scénario par Cathryn Humphries et Matthew Weiner, réalisation par Lesli Linka Glatter) - de

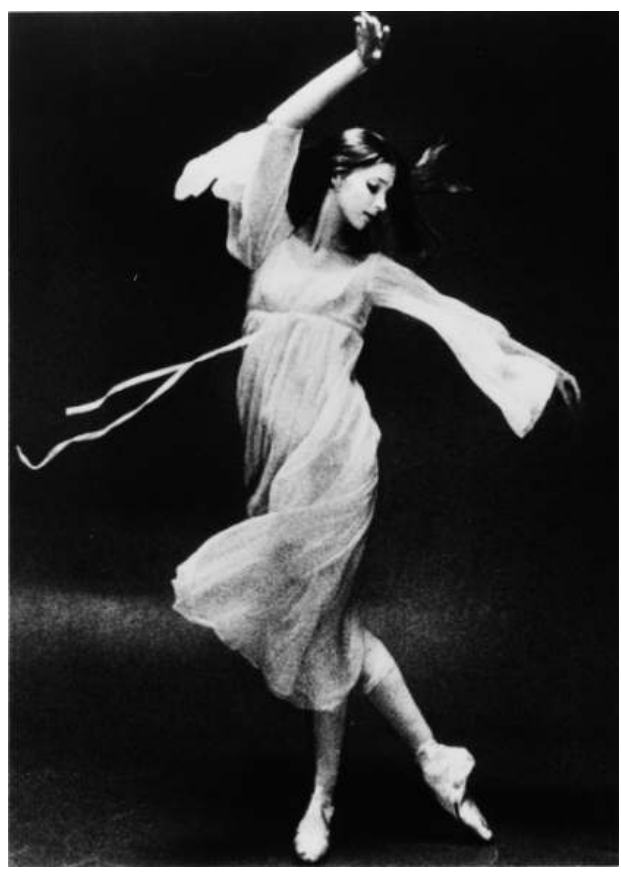
Mad Men, série pour la télévision créée par Matthew Weiner (déjà producteur de Sopranos) et produite par la chaine américaine AMC, qui a obtenu plusieurs prix. Je me limiterai à proposer quelques gloses sur cette poignée d'images en mouvement assez commerciales et mainstream, et à lancer quelques coups de sonde autour, avant et après elles, que j'ose prendre ici en tant qu'exemplification presque aporétique.

2 Pourquoi ? Je dirais, pour introduire la difficulté de mon sujet et pour me débarrasser d'un premier arrière-plan méthodologique non moins complexe, parce que ces quelques images sont doublement problématiques et glissantes. D'une part, il s'agit d'une exemplification qui défie toute condition symbolique d'exemplarité iconographique et iconologique ou pragmatique, c'est-à-dire qui résiste à être prise comme exemple probant, modélisant et modélisé, par récurrence ou exception, dérivation ou re-contextualisation etc., d'une quelconque typologie diachronique ou synchronique identifiable et lisible dans une tradition solidifiée ou cristallisée d'usages représentationnels. D'autre part, cette séquence conteste également, il me semble, les propriétés et les qualités esthétiques qu'elle devrait justement exemplifier, ou, si l'on préfère, qu'elle pourrait rendre à plusieurs titres accessibles et intelligibles. Selon cette seconde perspective, inspirée largement par Goodman, l'exemplification devrait en fait toujours impliquer une référence et une dénotation et les deux devraient être, au moins en principe, discernables et explicables. L'exemple choisi par le philosophe américain, qui est précisément traité par la fiction filmique qui m'intéresse, est la danse classique : ses motifs et ses figures, selon Goodman, « ce qu'ils exemplifient, ce ne sont pas des activités stéréotypées, habituelles ou familières, mais plutôt des formes dynamiques. Les motifs et les propriétés exemplifiés peuvent réorganiser l'expérience en mettant en rapport des actions qu'on n'associe pas habituellement ou en distinguant d'autres généralement non différenciées, enrichissant donc l'allusion ou affinant la discrimination $»^{1}$.

3 La plasticité de l'exemplarité d'une danse classique, ou généralement d'une danse assez strictement codifiée par un système notationnel, est donc souverainement esthétique, 
c'est-à-dire sensible et sensorielle, car elle touche à l'incorporation et aux actions et aux réactions naturelles du corps propre. Or, les actes d'un corps dansant, justement en tant que naturels et arbitraires, semblent pourtant faire abstraction des nombreux régimes d'historicité et de sémioticité malgré tout implicites dans les exemplifications elles-mêmes et dans les médias et les supports où elles se manifestent, ici comme gestes et actions de danse.

4 Nous voilà finalement face à deux aspects en tension, sinon en contradiction, qu'il faudra remettre dialectiquement au travail lorsque nous allons revoir et analyser les images en mouvement de notre petite séquence télévisée : d'un coté, l'exemplarité fait signe vers une dimension historique et temporelle complexe et impure, tantôt des systèmes symboliques singuliers, tantôt de leurs réalisations concrètes grâce à des média - notamment excédant les domaines des arts et de l'histoires de l'art et liés aux champs des arts populaires et des gestes du monde de la vie ordinaire - ; de l'autre coté, elle nous signale une a-historicité plus originaire, celle de la temporalisation de l'expérience esthétique primaire et fondamentale, c'est-à-dire corporelle et physiologique, pré-intentionnelle et pré-linguistique, notamment motrice. C'est précisément cette a-historicité trans-culturelle sous-jacente à toute complexification symbolique successive, y compris toute technique du corps, qui est aujourd'hui l'enjeu indépassable et majeur du débat sur les échanges possibles entre traditions et pratiques de savoirs très différents, telles que la neuro-phénoménologie et les sciences cognitives appliquées à l'histoire de l'art, l'anthropologie des images et l'esthétique.

\section{Vera incessu patuit Dea}

5 Sonder des pistes de ce dialogue, plus nécessaire et téméraire que jamais, voilà ce que nous invite à faire la petite séquence de Mad Men. Déjà le lieu de notre regard, spectateurs extérieurs, y est stratégiquement impliqué et engagé. Par un jeu très classique de montage alterné de plan et contre-plan, légèrement déplacés derrière le dos d'un corps détendu et élégant parmi les sujets de regard inscrits dans la scène, nous sommes commodément délégués d'une vision stabilisée et immobilisée, comme orientée à partir d'une figure confortablement installée dans une chaise-longue pliable ou le fauteuil d'une salle de cinéma, ou, pourquoi pas, d'un laboratoire de psychologie cognitive. Par ce dispositif spectatoriel, nous aussi assistons donc à une mise en scène non-professionnelle, commandée et partagée par une communauté assez restreinte, bourgeoise, mid-class et wasp. Allons prendre part à ce partage du sensible et à ses horizons d'attente et remarquons, pour commencer en bonne iconologie standardisée, quelques traits d'un premier régime d'historicité des images.

Le succès du spectacle ne réside certainement pas, pour le dire avec Jean-Pierre Changeux ${ }^{2}$, dans les " mêmes culturels ", c'est-à-dire dans les mémoires culturelles, non conscientes et à long terme, des spectateurs, assez distraits et bavards et pas forcement à jour des sources historiques, mythologiques et anthropologiques de la fête pour l'équinoxe de Printemps, dès Mystères d'Éleusis aux offrandes à Cybèle, aux divinités celtiques, et ainsi de suite. Personne, dans notre petite communauté esthétique du dimanche, ne connaît ou ne se souvient de la signification érudite de la répétition au cœur de la réapparition du Printemps, qui excède la chronologie progressive du calendrier et affirme une temporalité symbolique de la physis, une rythmique cyclique de la vie et de la mort - comme le retour vivifiant mais jamais 
définitif de Perséphone du froid Règne des ombres. Quoi qu'il en soit, la suite de la mise en scène de gestes et d'actions du corps au parc newyorkais n'est instituée ni par une quelconque forme de savoir préalable chez les spectateurs, ni par des techniques culturelles du corps chez les danseurs. Et pourtant, malgré cette prédétermination et prédestination des émotions pendant l'intervalle très prosaïque d'un weekend en famille, malgré cet enthymème affectif impuissant à activer une transfiguration du banal, écarté tantôt des sources profondes de la pragmatique rituelle, tantôt des palliatifs esthétisant des pratiques quotidiennes - malgré tout, quelque chose arrive. De l'imprévu, de l'inattendu. L'événement, comme disait Wittgenstein, arrive - comme quelqu'un qui met pied sur une scène ou entre dans une chambre. Quelque chose arrive donc. Non à un savoir de l'esprit, mais à un pouvoir du corps, non à un souvenir savant mais à un sentir désirant et ignorant.

7 Apparemment, donc, pas d'historicité, presque pas de culture. On pourrait dire, justement avec Nelson Goodman, ou Suzanne Langer, que le spectateur est touché par les sentiments - les feelings, c'est-à-dire les qualités expressives - des mouvements plutôt que par leurs formes; on pourrait ensuite ajouter, avec Rudolf Arnheim $^{3}$, que le mouvement est le plus fort attracteur et excitant visuel, et que cette surprise aspectuelle est déjà expressive. Chez le spectateur, réel ou fictionnel, pendant la vision d'un corps mouvant, réel ou en image, s'activent des potentiels d'action virtuelle qui n'ont rien à voir, en principe, avec son encyclopédie visuelle. Toute action transitive visant un but, et tout mouvement intransitif et expressif (c'est notamment le cas de notre petite danse), sont perçus par lui comme quelque chose de joué et d'agi par un objet biologique extérieur, vu ou imaginé par inférences. Selon cette perspective de lecture, qui est aujourd'hui celle de Vittorio Gallese ${ }^{4}$ et David Freedberg, la résonance corporelle du système-moteur de l'observateur est la condition neuronale minimale de toute possibilité de compréhension - même si cette compréhension commençante et aurorale de l'image et du corps, du corps de l'image, dont la neurobiologie empirique nous fournirait les corrélats localisables, n'a rien à voir avec le Verstehen de la psychologie existentielle et phénoménologique, ni avec Biswanger ou Maldiney.

8 A l'occasion du Spring reveal du 1963, il arrive donc au spectateur qui est notre délégué et lieutenant scopique fictionnel, d'être le support naturel d'une simulation incarnée ${ }^{5}$. Cette simulation préréflexive et immédiate se produit selon une logique du déplacement du désir et de la synecdoque de l'innervation organique, et se concrétise dans du presque-rien - rien d'autre que l'action minimale d'une main, sans but pragmatique ni souci artistique. Détaché du corps, ce geste presque hystérisé effectue une action très petite, très affaiblie et abrégée, en soi imperceptible et inconsciente, inadressée et pourtant emphatisée pour nous grâce au zoom impersonnel de la caméra, corrélat de cet autre zoom sur les détails anatomiques mis en exergue par un regard double, du protagoniste et du nôtre. Certes, sur le plan strictement diégétique, le geste mimétique non-intentionnel du spectateur révèle une imminence et annonce une suite, car il condense les liaisons pathémiques et les dynamiques existentielles des personnages : le protagoniste, Don Draper (John Hamm), Directeur créatif de l'Agence publicitaire Sterling Cooper Draper Pryce de New York City, va tomber amoureux de la jeune femme dansant, Suzanne Farrell (Abigail Spencer). Et pourtant, la Flora du Spring renewal est plus qu'une apparition circonstancielle limitée au contexte diégétique ${ }^{6}$. Elle est aussi une réapparition, l'instant d'une présentation sensible d'une forme qui se répète, qui se répercute entre des corps et des images, entre des corps en image et le corps du spectateur externe - le mien, en l'occurrence. Elle est l'exemplification d'une 
idée, au sens de l'eidòs, c'est-à-dire de la forme formatrice, qui structure les mouvements en figures de danse. "La danseuse n'est pas une femme qui danse », disait Mallarmé ${ }^{7}$. Non, elle n'est pas une femme qui danse parce qu'elle est en même temps l'arrivée incarnée, pour ainsi dire la revenue ou la survenue à une manifestation sensible et corporelle, de l'idée ou de la forme au sens grec : elle est l'exemplification passagère et imparfaite des figures récurrentes - des skhémata - d'une danse, des motifs réitérables d'une loi expressive dynamique. Elle est l'apparition-disparition des lieux communs cinétiques bien plus qu'iconiques, elle est l'incorporation mouvante des kinoi topoi de l'idée. C'est justement pour cela que cette forme universelle singulière est, quant à elle, partagée par la désindividualisation et la dépersonnalisation, entre corps individuels et individués, entre êtres distants et séparés - pour nous, la jeune méconnue Suzanne Farrell, et Flora, la Nymphe, origine non-historique pourtant visible et objet de désir, idée qui produit ses supports et scènes, forme immatérielle qui crée ses occasions et circonstances d'incarnation et d'apparition.

9 Je dirais que les propriétés esthétiques exemplifiées par la danseuse de l'épisode de Mad Men sont non localisables et participées, c'est-à-dire que les qualités à même de produire des effets sensibles sur le spectateur - intérieur et extérieur à la fiction surviennent d'une manière indécidable tantôt aux figures expressives du corps en mouvement et aux iconographies et chorégraphies implicites dans ses gestes, d'un coté, tantôt à une manifestation morphologique excédant la simple narration et ouvrant sur une figuralité cinétique abstraite, qui dénoue, il me semble, une mémoire enfouie des images comme telles - qui sont existentiellement là, indexicalement et indéniablement contemporaines - et une archéologie cryptée du médium lui-même, de l'autre. Et pourtant, la fictionalisation filmique semble oblitérer cet entrecroisement des régimes d'historicité profonde des images, des média et des effets, car - selon la leçon de Warburg et des théories de l'empathie reprises par ailleurs de façon assez problématique en neuro-esthétique - elle touche aux affects parmi les plus fondamentaux, notamment la puissance motrice élémentaire du Moi spectatoriel. Son corps est ainsi le lieu organique d'une subjectivisation érotique et esthétique par imitation musculaire ${ }^{8}$, par laquelle le spectateur se singularise par rapport aux effets prévus par le contexte prosaïque de la contemplation du spectacle et ouvre une nouvelle ligne de fuite au récit. Regardant de loin la figure de la danseuse, ses mouvements souples et gracieux, les élans des pieds nus, légers et suspendus sur l'herbe fraiche, le spectateur est malgré lui incité par une auto-affection endo-cinétique au plaisir d'une virtualité musculaire. Le mouvant est le véritable foyer diégétique, d'abord d'aventures sensorielles minimales et circonscrites, notamment les réactions entero- et proprioceptives intensifiées et diffuses, littérales et latérales - le désir visuel préhensif du spectateur touche ailleurs et, transporté, il se déplace -, qui réalisent finalement une catachrèse sensorielle ${ }^{9}$ et figurale qu'il faudra voir de près.

Comme chez les chasseurs, la visée vigile du spectateur est dissimulée; ses lunettes noires d'aviateur militaire, commercialisées depuis 1958, sont comme les Kulturbrille, les « lunettes culturelles » dont se plaignaient Warburg et Boas justement parce qu'elles aveuglaient à force d'être imprégnées de la mémoire des images. «I can't stop thinking about you ", dira ensuite Don à Suzanne, et dans cet énoncé dicté par une obsession érotique on devra malgré tout faire résonner ce qui nous hante en tant que spectateurs extérieurs, on devrait y faire ressentir notre impossibilité à terminer avec les mouvances de la jeune fille fleurie et leur mémoire en acte. Malgré le récit télévisé et l'amnésie du diégétique superficiel, malgré la réaction irréfléchie et l'immédiateté du 
mimétique corporel, nous ne pouvons nous empêcher de penser à elle, nous n'arrivons pas à consommer une fois pour toutes la répétition des mouvements de Flora, à parfaire les performances et les re-présentations de la Nymphe du Printemps revenue en tant que flower child au début des années soixante et de la génération hippie. Nous n'y arrivons pas puisque, aussi affaiblie et avilie, aussi diminuée et défigurée, c'est bien la Nymphe qui revient sous nos yeux pendant une fête écolière, ennuyeuse et routinière, à jamais orpheline de toute forme d'art ou d'artialisation.

11 On pourrait réaliser une sorte d'épochè stratégique et mettre entre parenthèses les dimensions historiques et temporelles de l'image en mouvement : peu importe s'il s'agit ou non d'une citation d'un modèle iconographique factuel ou d'une remémoration exemplificatrice d'une idée morphologique présupposée et intelligible dans le cas fictif singulier - en imposant une forme particulière aux éléments choisis et en complexifiant leur cohérence diégétique interne et leur richesse sémantique. Bien sûr, les régimes historiques et temporels des images et des fictions, qu'ils soient construits ou inconscients, de leurs productions et réceptions, de leurs actions et activations, sont essentiels pour toute compréhension et interprétation du visuel et $d u$ sensible. Pourtant, opter d'abord pour leur mise en réserve - pour une mise en charge de leur puissance -, cela nous permettra peut-être de nous débarrasser d'une différence ontologique et descriptive trop rigide et fixe entre l'esthétique et l'artistique. Notre option herméneutique en faveur du partage expressif et affectif entre les gestes, les mouvances et les actions du monde de la vie et les mouvements et les actes représentés du monde de l'art, a pourtant une fonction euristique assez délimitée. Ce parti pris pour une herméneutique pathémique nous permettra en fait de discuter, grâce à l'extrait tiré de Mad Men, l'hypothèse récemment proposée, en termes neurobiologiques, quant à un «niveau basique de réaction aux images [...] essentiel pour saisir l'efficacité autant des images de tous les jours que des œuvres d'art. $»^{10}$

\section{Pulsations, rotations, fictions}

Des pieds païens remémorant l'antique de Suzanne, absorbée par son agir corporel intransitif, aux doigts assez machistes et captivés caressant l'herbe de Don, spectateur statique et pourtant touché au corps, ému, remué, mis en mouvement. Comment décrire cette trajectoire de l'image en mouvement et de l'image du mouvement à l'émouvoir et au mouvoir du spectateur, en même temps minimal et mimétique, abrégé et déplacé ? Comment en saisir les filiations et les lignes de fuite ? Comment refaire, réactiver et réarticuler l'orbite de cette expérience sensible, touchant de loin ce que Jennifer Barker appelle l'œil tactile? Comment en tirer une petite parabole ou une fable méthodologique?

13 Sans trop de peine, on peut dire que l'extrait de Mad Men met en scène une "empathie d'activité » [Tätigkeitseinfühlung]. L'expression est de Moritz Geiger, élève de Theodor Lipps et phénoménologue à Munich, tirée notamment d'une allocution au Congrès International de Psychologie Expérimentale de Innsbruck en 1910, louée par Husserl. Plus précisément, les pirouettes sur place des doigts du spectateur réalisent ce que Robert Vischer, dans son texte fondateur sur le sentiment optique de la forme (1873), avait appelé l'« émotion motrice indirecte » [motorische Nachfühlung] à partir d'une " émotion sensorielle directe » [sensitive Zufühlung], notamment visuelle. A propos de l'em-pathie, du pâtir-in (Einfühlung), et de l'en-ésthésie, du l'appréhension du sensible en une 
perception unitaire et intériorisée $»^{11}$, Vischer distinguait l'Affektswoll, l'empathie mimétique, ou agente, d'une empathie physionomique, Stimmungswoll, sans motilité, soit-elle contractée ou expansive par rapport à «l'empreinte d'un phénomène ", qui remplit passivement sentir-avec (Einempfindung), et de l' « unification des sensations qui organise le sujet ${ }^{12}$. Les mouvements décontractés, voire languides, des doigts du spectateur imitent les courbes souples et gracieuses des jambes et des pieds de la danseuse. Il s'agit, je le répète, d'une imitation immédiate, même si minimale et à peine au-delà du seuil du plaisir de l'entre-perception et de l'auto-affection sensori-motrice, et agente, puisqu'elle engage justement des mouvements non finalisés. Regarder, soit une action transitive et finalisée, soit un mouvement intransitif et expressif, et notamment un corps déplacé et hors équilibre, off balance, est déjà simuler : voir, c'est agir, c'est faire comme $e^{13}$. Or, cette imitation engagée automatiquement et immédiatement, en-deçà de l'acte délibéré et intentionnel de l'imagerie visuelle et motrice, mentale et réelle, est mesurée par une logique de la sensation qui est à part entière une logique temporelle, une logique du Nach, du « re-». Les micro-mouvements et les aperçus moteurs minimaux des doigts de notre spectateur fictionnel, sont en fait cadencés par une responsivité auto-poïétique similaire à celle de l'organisme biologique aux stimuli du milieu et qui pourtant implique structurellement le rejouer et le ressentir. Et leurs autonomisation presque hystérique, car si la main se porte souvent où nous ne la plaçons pas, comme disait déjà Montaigne non par hasard à propos de l'imagination ${ }^{14}$, les réactions délocalisées et virtuellement manipulatoires de la main du spectateur foudroyé sont en fait réglées par un double pas ou un battement rythmique, à la fois d'incorporation et de projection, de miniaturisation et de métonymie.

Il faut alors énucléer une polarité figurale de cette empathie mimétique et motrice indirecte, répétitive et ressouvenant. Il faut, avec Deleuze ${ }^{15}$, y relever une articulation structurelle et génétique entre l'imprégnation (végétale, passive) par forces, expressions et qualités incarnées dans un état de choses, une ambiance ou un milieu, d'un coté, et l'extension (animale, active) d'actions ou d'équivalents d'actions, de l'autre. Par là, on pourra contester les objections qui ont été faites, à tort dirais-je, à l'empathie. L'Einfühlung, en fait, n'est pas seulement extro-flexion, projection et injection d'un potentiel imaginaire dans le perçu; elle n'est pas, non plus, une sorte de volonté de puissance et d'intensification hypertrophique de la sensation vitale générale, d'englobement de part du pole subjectif du pole objectal, totalement dévalorisé, qu'il soit inerte ou vivant ${ }^{16}$. Certes, c'est la version fort réductrice adoptée par quelques travaux de neuro-cognitivistes qui s'occupent d'esthétique et qui parfois donnent l'impression de confondre le physique et le phénoménologique, les aspects et les interprétations, les modèles et les théories ${ }^{17}$. Sans oublier les distinctions introduites par Robert Vischer et Theodor Lipps ${ }^{18}$, ensuite reprises via Goethe par Warburg, on devra au contraire garder la polarité qui structure l'empathie et sa figuralité incarnée, sa dualité et, dirais-je, l'indécidabilité qui règle la formationnégociation de tout devenir-geste des affects.

15 Regardons encore une fois les doigts vagabonds de notre spectateur, leurs mouvements à peine esquissés, ces engrammes inscrits dans le support mouvant et incommensurable de l'herbe - ce singulier collectif pour la matière elle-même, cet autre nom de l'hylétique jamais identique à lui-même. Ces écritures sans scribe sont en même temps imitation ou simulation incorporée des mouvements d'un autre corps perçu, et expression ou performativité projective de l'auto-affection du corps propre du spectateur. Ces petits skhémata tracées à fleur de peau sont à la fois mimémata et 
pathémata en acte, résonances endo-cinétiques de l'autre et réfractions cryptopréhensives sur et autour de lui. Vischer ${ }^{19}$ distingue, dans la " vie représentative " [Vorstellungsleben], précisément la stimulation constante et la pulsation, caractéristique de l'émotion sensorielle directe, de la rotation successive, enveloppante et flottante [Umschweifen, Umschmiegen und Bestreicheln], déterminant l'émotion responsive indirecte. La profondeur implicite dans ces contournements et ces détours virtuels esquissés par la sensation efférente, est, en fait, le noyau le plus élémentaire et primitif de l'angoisse pour la tridimensionnalité plastique, anxiété perceptive et donc existentielle emphatisée par Riegl et Worringer et décisive ensuite chez Panofsky pour déterminer les performances du revolving view du spectateur face à la figura serpentinata. Les mouvements élémentaires des doigts de la main du spectateur empathiquement engagé sont en même temps caresse et écho, l'une et l'autre, ni l'une ni l'autre, elles incarnent le neutre ou l'entre-deux, l'enjeu polaire entre actif et passif. Leurs glissements enjambés donnent lieu à une réversibilité (mimétique) entre palpation et hospitalité, entre une direction centrifuge - qui va du sujet vers le mouvant extérieur -, et une direction centripète - qui va de l'objet mobile vers la motilité intérieure, autoaffectée et viscérale du sujet du regard.

Pour attraper au vif l'innervation responsive indirecte de l'émotion de l'œil par le mouvement de la main, c'est-à-dire pour prendre en flagrant délit le déplacement sans ambigüité du désir scopique envers l'organe préhensif par excellence, qui se porte famélique là où le sujet n'en sait rien et contourne et enveloppe son objet trop loin, je propose un contre-exemple. En position de spectateur éloigné et furtif, le regard de Rodolphe (Jacques Borel, alias Jacques Brunius) déguste le spectacle d'un autre corps de femme, qui s'offre en une sorte d'acmé rythmique, la jeune parisienne Henriette Dufour (Sylvia Bataille) à la balançoire, totalement absorbée par un " réalisme affectif déchirant ", comme l'appelait André Bazin ${ }^{20}$. Il s'agit d'une scène célèbre de Une Partie de campagne, court-métrage que Bazin définit justement "sublime » et " parfaitement terminé ", tourné par Jean Renoir en 1936 dans la forêt de Fontainebleau, sur les rives $\mathrm{du}$ Loing, près de Bourron-Marlotte, pays du père peintre et de Cézanne, d'après le conte de Maupassant (1881), avec la photographie (du tournage) de Eli Lotar et l'assistance à la régie de Cartier-Bresson et Luchino Visconti, montage définitif de Marguerite Houllè-Renoir (en 1946), avec la participation lumineuse de Georges Bataille. Nous sommes encore une fois au Printemps, et donc en bonne mémoire, sans doute picturale ${ }^{21}$, soit de Flora, soit du Faune. Et nous sommes éblouis par cette " comédie [qui] sans cesse se dissout dans l'émotion ${ }^{22}$ et qui nous montre le spectacle du Printemps lui-même, qui nous fait participer à la diffusion atmosphérique d'une " sorte de désir vague » entre les personnages, les choses et les êtres.

Emblème aléatoire, et pour cela souverain, de ce partage empathique sans sujet, bien au-delà de la projection subjective imputée à l'Einfühlung, est un papillon : pendant le dialogue entre la mère (Jeanne Marken) et la fille, précisément sur le Printemps et le désir, le minuscule lépidoptère ne cesse de voltiger entre elles, incarnation de l'événement éphémère du réel à la limite de la visibilité au sein du feuillage frémissant, élément pro-filmique qui entre et sort, contingent et erratique, du cadre. Cet événement rythmique excessif et infinitésimale semble miniaturiser après-coup la pulsation des apparitions et des disparitions, captées par un cadre fixe et en contreplongée, du corps joyeux et panique de la jeune femme, volante ou volage - comme on 
voudra -, femme-oiseau ou femme-papillon, clignotant sur le fond tantôt du vide du ciel, tantôt du plein de la sylve.

Cette fois, la formule expressive du geste du spectateur-voyeur n'est embarrassée par aucune polarité entre actif et passif ; le regard empathique et explicitement érotisé est nettement projectif. Les micro-rotations des doigts de notre deuxième lieutenant scopique fictionnel sont expressément sadiques - et fort ridicules -, occupées comme elles sont à simuler et anticiper une prise du corps en tant qu'objet d'empathie; sauf qu'ici le suffixe en/em dit une véritable pénétration du Même dans l'Autre ${ }^{23}$.

Par ailleurs, l'érotisation était déjà assez remarquable et connotée tactilement chez Vischer $^{24}$ (comme ensuite dans le voir-selon de Merleau-Ponty), lorsqu'il dit que « nous nous mouvons avec et dans les formes [in und an den Formen] " et " de nos mains aimantes, nous cherchons à toucher [tasten] toute modification de l'espace »

\section{Illusions et fantômes}

Selon Vischer, c'est justement le contact entre deux mains qui illustre parfaitement la perméabilité entre l'affecté et l'affectant, le touché et le touchant. Il s'agit d'un point parmi les plus délicats et controversés de la logique sensible de l'empathie, concernant l'articulation même des polarités entre actif/passif, projection/incorporation, subjectivation/altération, identité/étrangeté, et ainsi de suite. Vischer ${ }^{25}$ écrit :

"Je me fie à la forme inanimée [der leblose Form] de ma vie individuelle, de même que je crois à bon droit à un non-moi [Nichtich] vivant et personnel. Je me garde donc qu'en apparence moi-même, bien que l'objet demeure un autre. Je semble m'accommoder et d'adapter à lui comme la main à la main et pourtant je suis secrètement transporté et ensorcelé dans ce non-moi. [Ich scheine ich ihm nur auzubequemen und anzufügen, wie Hand im Hand sich fügt und dennoch bin ich heimlicher Weise in dieses Nichtich versetzt und verzaubert] »

21 Cette réversibilité, ensuite longuement travaillée par la phénoménologie de la perception en dialogue continuel avec l'anthropologie et la psychologie profonde du magique, est à mes yeux au cœur du chiasme haptique mis en scène dans la séquence de Mad Men qui nous occupe.

La main de notre spectateur interne, lorsqu'il quitte le monde circonscrit des ustensiles et des actions orientées vers un but - le verre qui apaise sa soif ou son ennui - et désactive les conduites sensori-motrices finalisées, se déterritorialise en organesmatières d'expression, en tropes-supports d'affect. Ses doigts incarnent et exposent des qualités, inchoatives et indécidables, entre imitation et appropriation, entre réception et émulation du mouvement et usurpation du mouvant, entre résonance de la danse, au-delà de la danseuse, et caresse de son corps, rêverie de palpation de l'incarnation chancelant de l'idée... Les mouvements molécularisés des doigts de notre lieutenant scopique face à la danse de l'image et en image, à son aller et retour par élans et retenues, ne sont pas, à la rigueur, des actions proprement dites : ils sont à l'action ce que la danse est à la marche. Je n'évoque pas ici la distinction introduite par l'« ontologie motrice » de la nouvelle philosophie neurobiologique de l'action, mais une équation de Valéry ${ }^{26}$ :

«C'est [...] bien que la danseuse est dans un autre monde, qui n'est plus celui qui se peint de nos regards, mais celui qu'elle tisse de ses pas et construit de ses gestes. Mais, dans ce monde-là, il n'y a point de but extérieur aux actes; il n'y a pas d'objet à saisir, à rejoindre ou à repousser ou à fuir, un objet qui termine exactement une 
action et donne aux mouvements, d'abord, une direction et une coordination extérieures, et ensuite une conclusion nette et certaine. » engendrée par un captage visuel ? La plasticité des représentations intermodales des parties du corps et de l'espace personnel et péripersonnel, c'est-à-dire de l'espace délimité par la distance de préhension, a été illustrée très tôt. Aristote ${ }^{27}$, commenté dans la Dioptrique par Descartes, le suggérait déjà en invitant le lecteur à tenir une boule entre l'index et le majeur, puis à fermer les yeux, croiser les doigts et y glisser à nouveau la boule : la sensation de contact avec la boule est ressentie non au point réel de contact, mais, doublée, à l'extrémité des doigts, là où la sensation se manifeste habituellement. On conclura que l'espace péripersonnel et le schéma corporel se modifient et s'étendent en fonction des objets extérieurs qu'on touche réellement ou, et c'est décisif, en fonction des affections, en même temps endogènes et allogènes, produites par incorporation et projection empathique à distance.

Aristote confirme ce qu'il avait déjà soutenu ailleurs : il y a une relation très puissante, et pourtant tout à fait habituelle, entre, d'un coté, les sensations et les passions, aisthémata et pathémata, et, de l'autre, les images comme illusions (phantasmata) ou comme altérations sensorielles du rapport entre le senti et le perçu. Les images sont l'effet des sensations qui demeurent sensibles comme telles, tantôt quand l'objet perçu est absent, tantôt quand, tout étant donné en présence, les apperceptions se mélangent aux percepts. Dans cet entrecroisement fantasmé, est au travail une fonction d'incorporation et de vicariat réciproque des sens ${ }^{28}$, c'est-à-dire une fonction poïétique, modale, active et mouvante, selon laquelle les contenus perceptifs se concrétisent et se différencient par rapport aux diverses régions sensorielles. Or, il faut remarquer que, ce cadre physio-phénoménologique donné, Aristote évoque les erreurs et les illusions qui arrivent à nous tous alors que nous sommes passionnés [en tois pàthesin òntes]. Parmi les êtres affectés par les désirs les plus puissants [epithumiai], il cite le lâche et l'amoureux, victimes de la peur et du désir, transportés, "à la suite d'une petite ressemblance » et de la moindre similitude, par les illusions, par les fantasmes : la « moindre similitude fait d'autant plus apparaître ces illusions qu'on est davantage sous le coup de la passion ". Aristote évoque également le malade en accès de fièvre et le rêveur, qui croient voir des lézards sur le mur à la place des zigzags des craquelures et font même des mouvements, extérieurs ou endogènes, réels ou imperceptibles, vers ces apparitions, pour toucher ces phantasmata fugitives.

Tous les éléments mis en lumière par Aristote, pris entre physiologie de la perception, du rêve et de la phantasia et psychopathologie de la vie ordinaire et amoureuse avant la lettre (et pour cela destinés à la plus grande fortune philosophique et interprétative), tous ces éléments se cristallisent dans une œuvre de Max Ernst. Au premier mot liquide ${ }^{29}$ fût réalisé en 1923 comme décoration de la chambre de Cécile, fille de Paul et Gala Eluard, à la maison d'Eaubonne. L'hypo-texte ${ }^{30}$ de cette peinture pariétale est une illustration, publiée en 1881 dans la revue scientifique à large diffusion La nature, fondée en 1873 par Gaston Tissandier. C'était l'illustration d'un article sur les illusions, précisément l'illusion d'Aristote que je viens d'évoquer. Les lignes entrecroisées des doigts, fourchette gracieuse et languide, dessinent le X du mystère du sexe féminin, incarnent un corps suspendu, sans poids, entre terre et air - un corps-nuage, un corpsdanse. C'est le corps ascensionnel et fluctuant de La puberté proche (1921), flamboyant

Images Re-vues, Hors-série 4 | 2013 
Übermalung, glorieuse méta- ou trans-peinture qui se répercutera dans le collage-récit $L a$ femme 100 têtes du 1929.

Comme le X tracé par les lézards sur la façade menaçant du début de la Maison du chat qui pelote de Balzac, signe fortuit qui donne « aux historiens la facilité de reconstruire par analogie l'ancien Paris », le reptile au mur «bariolé d'hiéroglyphes » de Ernst interpelle l'interprétation. A condition qu'elle soit incarnée, vécue, pathémique, voire engagée, bref existentielle. Signe statique en mouvement apparent - comme les lézardes pour les rêveurs, les fébriles et les amants énervés d'Aristote, et comme «les ébauches ou les traces [Ansatze und Spüren] d'attitudes, d'excitations ", d'un " secret tressaillement [...] à peine réprimé, de l'élan et de la crainte ", pour les passionnés empathiques de Vischer ${ }^{31}$ : pour nous tous donc -, la forme reptilienne de la peinture d'Ernst entraine un changement génétique et finalement moteur du sujet qui regarde, il emporte son corps à un changement de lieu et, à la limite, de nature. Vischer ${ }^{32}$ est sur ce point essentiel très clair :

«En un éclair, ces signes sont traduits en leur signification comportementale correspondante. [...] La mimique expressive est intérieurement exécutée et répétée : la forme stable est donc ressentie en elle-même comme si elle était en mouvement manifeste. »

27 Mais il faut faire attention: la traduction, l'übersetzung, et son immédiateté foudroyante, Blitzschnell, décrit ici précisément le mouvement de la connaissance à travers l'émotion, le transport de la signification par le biais de la passion, bref l'incorporation du sens - l'être, ou plutôt le devenir ou la métaphorisation somatique du sémantique. Si toute description d'une œuvre d'art visuel, "avant même de commencer », comme écrit Panofsky dans un texte fondamental, « aurait dû inverser la signification des facteurs de représentation purement formels pour en faire des symboles de quelque chose qui est représenté $"^{33}$, cette incessante opération de traduction, inversion, substitution, à savoir cette abstraction complexe, est mise en place face au visible en général, et pas forcément à un artefact, et, surtout, elle est d'abord affaire d'une poiétique du corps. "La signification des corps chasse leur forme ", comme dit Valéry, et nous tous, certes avec nos compétences et expériences, différentes et singulières, percevons un certain système de phénomènes, mais chacun de nous sur-le-champ les "transforme en signes, qui [nous] parlent à l'esprit comme feraient les teintes conventionnelles d'une carte. Ces jaunes, ces bleus, ces gris assemblés si bizarrement s'évanouissent dans l'instant même ; le souvenir chasse le présent ; l'utile chasse le réel [N]ous ne voyons que du futur ou du passé, mais point les taches de l'instant pur. [...] Rien, peut-être, de plus abstrait que ce qui est. $~^{34}$

Inspiré, parmi d'autres choses, par la fortune iconographie et interprétative du thème de l'illusion tactile et de ses rapports avec la puissance cinétique des phantasmata du désir et du rêve mise à jour par Aristote, le lézard-lézarde au mur de Au premier mot liquide emblématise ainsi le pathos moteur et le désir de toucher inhérents à l'apparition même de l'image, à sa figurabilité presque cinématique ${ }^{35}$. Ernst, suivant de très près Aristote, parle très précisément de « la persistance et la rapidité qui sont le propre des souvenirs amoureux ». Par ailleurs, le lézard au mur peint en style pompéien paraît emprunté à un récit de rêve de la Gradiva ${ }^{36}$ de Jensen, commentée par Freud, et il nous semble la synecdoque figurative de l'apparition de l'allure flottante de la Nymphe au pied léger et dansant. 


\section{Plus rien du tout, rien que du vent}

29 A lire le traité d'Aristote sur le rêve, tout attracteur visuel - illusoire ou réel, instable ou objectal - est encore opérateur de conversion tactile et d'action sensori-motrice, même si virtuelle et déplacée, ou diffusée. Ce point capital ouvre une tradition millénaire et n'a pas échappé aux chercheurs les plus attentifs qui travaillent aujourd'hui sur les corrélats neuronaux de l'empathie esthétique.

Un article publié par l'équipe de Gallese affirme que l'espace environnant est plein d'objets qui s'entre-touchent de manière accidentelle, sans impliquer aucun engagement humain, aucune source animée ni vivante. Autrement dit, et d'ailleurs selon nos expériences les plus ordinaires, l'on peut être ému observant un arbre s'abattre sur un banc de parc, les feuilles chatouillées par le vent, des gouttes d'eau qui retombent sur les feuilles d'une plante, la surface d'eau morte d'une flaque agitée par une averse d'été. Ni l'actant, ni le support biologique d'une action ou d'une expression proprement dites, ne sont donc nécessaires et indispensables pour qu'il y ait une expérience d'émotion esthétique, lato sensu. Les auteurs parlent de " témoignage du toucher dans la nature ", de son événement impersonnel qui n'appartient à personne.

Or, reprenons-nous à la lettre cette description expérimentale pour analyser une dernière fois la petite séquence de la sitcom américaine.

La caméra fixe suit les mouvements gracieux et souples de la danseuse, s'adapte à sa trajectoire circulaire, qui tourne autour de l'axe en bois comme un carrousel. Et pourtant, détachée du point de vue pseudo-subjectif du spectateur d'abord partagé, la caméra ne s'arrêtera pas sur le corps de la femme, résistant à la poussée de l'élan contre le poids, mortel, de la gravité. Au contraire la caméra abandonne le corps dansant, retombé au sol immobile, au bord du champ visuel et suit, en haut à droite, la ligne de fuite centrifuge, irrésistible, du mouvement spiralée, pour finir dans un fondu frétillant avant le cut. $\mathrm{Ni}$ espace naturel, où la vision existentielle et pathémique s'ignore, ni espace artificiel, où son désir empathique se narrativise, le brouillement visuel, où s'achève le trajet de la caméra, n'est finalement que l'indice d'un manque. A moins d'y pressentir non pas le dévoilement possible, de la part d'une action ou d'un geste, d'une situation qui n'était pas donnée, mais la présentation sensible de l'idée esthétique immanente au percept. L'effet de pan des feuilles agitées qui clôt la séquence de la danse est plutôt de l'ordre d'une délivrance de l'affect et d'une empathie tactile abstraite, que d'une liaison diégétique instituée par l'aspect, ou d'une focalisation à visée exemplificatrice de ses propriétés esthétiques.

En d'autres termes, le mouvement de la caméra, impersonnel et expressif, dessine d'abord une ligne de force tangentielle en esquissant un vecteur diagonal et centrifuge. Ensuite, elle se déplace et s'éparpille dans les feuilles animées par le vent, indices innombrables de l'external non biological force dont parle Vittorio Gallese ainsi que de la breeze from Wonderland qui souffle sur les plis de la robe de Lolita de Nabokov et de l'aüssere Veranlassung di Warburg. Or, il faut souligner que la manifestation de cette vis motrix ${ }^{37}$ non-biologique, insaisissable mais tactile, aérienne mais incarnée dans le " frémissement éternel des feuilles "(Andrea Zanzotto), dépasse toute différence iconique, tout cadrage perceptif et toute focalisation aspectuelle en forme de fondfigure ${ }^{38}$. 

majeurs de la Gestaltphychologie, avait mis l'accent sur une expressivité de l'inanimé totalement irréductible aux théories faussement simplistes et hyper-subjectivistes de l'« inférence pathémique» du pathetic fallacy, critiqué en tant que principe associationiste d'une similarité structurelle entre sujet et objet totalement fondée sur la projection d'un état mental ou sentimental du spectateur sur un corrélat aspectuel et physique du perçu. En revanche, l'expression est déjà, pour Arnheim, le contenu primitif et originaire de toute perception : expressive est la qualité physionomique des forces agissantes dans le milieu environnant, c'est-à-dire dans l'espace anthropologique en général - forces qui sont, non par hasard, exemplifiées plutôt par des flammes spiralées, des feuilles tourbillonnées, des murs lézardés, que par des artefacts. C'est justement cette qualité sensible et expressive qui engage les dynamiques sensorielles, émotionnelles et cognitives du spectateur. Déjà Merleau-Ponty ${ }^{40}$, après Biswanger, Minkowski et Cassirer, avait dit l'essentiel : toute « « apparition » [Erscheinung], est une incarnation [Verkörperung] et les êtres ne sont pas tant définis par des "propriétés " que par des caractères physionomiques. [...] Les choses sont prises pour l'incarnation de ce qu'elles expriment, que leur signification humaine s'écrase en elles et s'offre à la lettre comme ce qu'elles veulent dire. Une ombre qui passe, le craquement d'un arbre ont un sens: il y a partout des avertissements sans personne qui avertisse. »

A la suite d'Arnheim et de Merleau-Ponty, nous pouvons mieux comprendre que l'expressivité du visuel et du perceptible en général s'incarne dans des épiphénomènes indexicaux ou tactiles, tels que les feuilles agitées de Mad Men, ou de Le repas de bébé des frères Lumières, en 1895. Elle échappe à toute conceptualisation intellectuelle, à toute détermination objective de ses propriétés, causes et effets, tout en affectant, malgré cela, le corps et la pensée, la motricité et le mouvement de la réflexion. Donc, au-delà de toute hypothèse intertextuelle, nous devons y voir une empathie abstraite et une incarnation de l'idée esthétique au sens kantien. Le lieu amorphe et moléculaire du mouvement, sur quoi le regard innervé et tactile du spectateur de Mad Men se consume, est le milieu d'immanence expressive et figurale pour une subjectivisation impersonnelle. Et, pour cela, il est lieu commun pour d'autres images, d'autres affects. Au sein du fond continu, hylétique, du tourbillon des feuilles mues par le vent rugueux (Pierre Reverdy), aucune différence iconique est détectée, aucun aspect est dégagé, aucune est figure détachée : les données perçues perdent leur organisation et individualité formelle ${ }^{41}$. Par cette présentation sensible, par cette «incorporation visuelle de l'idée» ${ }^{42}$, quelque chose nous est encore donné à penser. Inhérent au regard expressif de la caméra, notre regard prend donc position ${ }^{43}$ et touche finalement au mouvement et au rythme inhumain, abstrait, de tout corps ainsi que toute image, " victime, comme disait Mallarmé ${ }^{44}$, d'une impuissance extatique à disparaître » dans la danse de toute chose.

De l'action a-téléologique du corps de la danseuse, intensifiée en gestes expressifs et en détails anatomiques, à l'action-imitation minimale des doigts du spectateur, désœuvrée en échos crypto- et endo-cinétiques et petites auto-affections, jusqu'à l'expression déindividualisée du milieu arborescent, actualisée en petites images-perceptions indexicales en formation sans corrélat objectaux : le mouvement de la caméra dessine une dé-particularisation des formes mouvantes et des supports biologiques dans un échange empathique général. Elle incarne une vocation empathique et abstraite du spectateur - tantôt intérieur, tantôt extérieur - à s'incorporer au mouvement des

Images Re-vues, Hors-série 4 | 2013 
mouvements. Le champ visuel quasiment saturé, tout terme identifiable d'une perception exprimée presque indiscernable mais pas encore disparu, la vision devient presque rapprochée, selon une "vertigineuse proximité " au noyau sensible, si fluctuant et contingent qu'il soit ${ }^{45}$. Avant de se dégager et se distancier de toute innervation motrice immédiate avec l'image et de revenir au récit, notre regard se fait fugace, allusif, soucieux des aspects instables et des textures éphémères du visuel. Il est peut-être absorbé par les qualités expressives et haptiques du support et du médium lui-même.

En principe et à la limite, il ne s'agit plus d'une simulation incarnée, directe ou par inférence, des corps, ni des êtres animés, humains inclus, ni des objets. Il s'agit plutôt d'une imitation corporelle, d'une incorporation tactile de loin du « corps du film » luimême ${ }^{46}$. Peut-être de l'idée du cinéma. Entrainée par le mouvement de la caméra, notre vision se fait, peut-être malgré nous, morphologique et haptique, régressive et archéologique, et touche de loin à la matière même, instable et moléculaire, pour ainsi dire «passée à transport " $^{47}$, de l'image sans phrases ${ }^{48}$.

C'est encore Arnheim qui nous en fournit la meilleure description : dans un article ${ }^{49} \mathrm{du}$ printemps 1963, comme la Maypole school dance de Mad Men, il la nomme « la jungle des phénomènes matériels»; avec Giambattista Vico, je la dirais plutôt l'ingens sylva du sensible. L'expression d'Arnheim est sans doute très négative, presque malveillante eu égard au grand livre américain sur le cinéma de Kracauer, Film (1960), mais il faut prendre au sérieux son exotisme involontaire. Dans la jungle du visuel, enregistrée en toute sa matérialité changeante et informe, on saisit l'indice historique de la nature au Vietnam, qui est en même temps l'extériorité absolue, voire l'Autre et le réel inassimilables, et justement pour cela singulièrement contemporaines. Plus radicalement, dans cette concomitance figurale entre la vibration des feuilles d'un arbre dans un parc new-yorkais et le fourmillement des feuillages enchevêtrés de la jungle vietnamienne, on surprend une image dialectique au sens de Benjamin ${ }^{50}$.

Finalement, on met à jour un symptôme assez important de toute théorie de la perception, notamment de la Gestalt, lorsqu'elle fait abstraction tantôt de la présentabilité du matériau sensoriel et expressif de l'existence comme telle en jeu dans l'Einfühlung, tantôt de la valeur profondément politique du figurable rejoint par régression empathique.

«L'existence » Sartre écrit dans La nausée ${ }^{51}$, «n'est pas quelque chose qui se laisse penser de loin : il faut que ça vous envahisse brusquement, que ça s'arrête sur vous, que ça pèse lourd sur votre cœur comme une grosse bête immobile - ou alors il n'y a plus rien du tout ». Il n'y avait plus rien du tout, j'avais les yeux vides et je m'enchantais de ma délivrance. Et puis, tout d'un coup, ça s'est mis à remuer devant mes yeux, des mouvements légers et incertains : le vent secouait la cime des arbres ».

\section{NOTES}

1. N. Goodman, Langages de l'art. Une approche de la théorie des symboles, tr. fr. J. Morizot, Hachette, Paris 2005, p. 96. 
2. J.-P. Changeux, « De la science vers l'art » (1988), Raison et plaisir, Odile Jacob, Paris 1992, pp. 58-61.

3. R. Arnheim, Art and visual Perception. Psychology of creative Eye, California University Press, Berkeley, London, Los Angeles 1974, p. 372.

4. Sur la bodily resonance, D. Freedberg, V. Gallese, « Motion, emotion and empathy in aesthetic experience », TRENDS in Cognitive Sciences, vol. 11, n. 5, May 2007, p. 197; cf. G. Rizzolatti, L. Fogassi, V. Gallese, «Neurophysiological mechanisms underlying the understanding and imitation of action », Nature Reviews in Neuroscience, vol. 2, n. 9, September 2001, p. 661.

5. Ibid.

6. Suzanne Farrell était, en réalité, une véritable danseuse professionnelle américaine, qui affirmait sa célébrité justement au début des années soixante dans le New York City Ballet de George Balanchine et qui fondera ensuite, au John F. Kennedy Center for the Performing Arts de Washington, un ballet très renommé, le Suzanne Farrell Ballet. Déjà le nom, donc, inscrit dans le corps du personnage de fiction une synchronie légendaire avec la danse contemporaine nord-américaine.

7. S. Mallarmé, « Ballets » (1886), Euvres complètes, éd. H. Mondor et G. Jean-Aubry, Gallimard, Paris 1959, p. 304: « A savoir que la danseuse n’est pas une femme qui danse, pour ces motifs juxtaposés qu'elle n'est pas une femme, mais une métaphore résumant un des aspects élémentaires de notre forme, glaive, coupe, fleur etc., et qu'elle ne danse pas, suggérant, par le prodige de raccourcis ou d'élans, avec une écriture corporelle. »

8. S. Schaviro, The Cinematic Body, The University of Minnesota Press, Minneapolis 1993, p. 52 sq, J. Barker, Tactile Ey: Touch and cinematic experience, California University Press, Berkeley - Los Angeles - London 2009, p. 73-82. Pour une reconstruction génèrale, M. Tausing, Mimesis and Alterity: A particular History of the Senses, Routledge, New York 1992.

9. V. Sobchack, Carnal Thoughts: Embodiment and moving Image Culture, University of California Press, Berkeley - Los Angeles - London 2004, pp, 76 sq.

10. D. Freedberg, V. Gallese, « Motion, emotion and empathy in aesthetic experience », loc. cit.

11. R. Vischer, Du sentiment optique de la forme, trad. fr. par Maurice Elie, préface de Carole Talon-Hugon, dans Aux origines de l'empathie, Fondaments \& fondateurs, Ovadia, Nice 2009, p. 77 sq. Cf. S. Caliandro, «Empathie et esthésie un retour aux origines esthétiques », Revue française de psychanalyse, vol. 68, n. 3, 2004, note 7 .

12. A relire selon la filiation Semon-Warburg et Peirce-Deleuze, l'empreinte est lien émotionnel et sensori-moteur entre objet, ou situation, et sujet (acteur et spectateur). Cf. G. Deleuze, Image-mouvement I, Minuit, Paris 1983, p. 218-9.

13. Cf. V. Gallese, « Embodied Simulation: From neurons to Phenomenal Experience », Phenomenology and the Cognitive Sciences, vol. 4, n. 4, December 2005, p. 34-6.

14. Montaigne, Les Essais, I, 21.

15. G. Deleuze, Image-mouvement I, cit., pp. 214-9. Cf. J. Gil, « La danse, le corps, l'inconscient », terrain, n. 35, septembre 2000, p. 57-74, et Ouvrir le corps, in Lygia Clark, de l'œeuvre à l'événement, Musée des Beaux Arts de Nantes, Nantes 2005, [s.p.]

16. Je note au passage que on est assez proche à la « carnal rhetoric of identification» du fétichisme selon Willian Pietz: cf. W. Pietz, « The Problem of the Fetish I », RES, n. 9, Spring 1985, p. 14.

17. L. Pizzo Russo, So quel che senti. Neuroni specchio, arte ed empatia, ETS, Pisa 2009, pp. 33, 46, 51 sq.

18. Qui, dans son Esthétique distinguera quatre types d'empathie ${ }^{: 11}$ la première, dite « empathie aperceptive générale ", est une tendance humaine à projeter de la vie dans les formes ; 2) la deuxième, dite empathie empirique, est la réalisation de cette tendance générale en relation avec une forme donnée qui la détermine; 3) la troisième, dite " empathie d'états d'âme ou de tonalités émotives [Stimmungseinfühlung] ", est l'état psychique correspondant au caractère de la forme individuelle ou de l'atmosphère aperçue et vécue ; 4) la quatrième, est l'empathie avec autrui, intersubjective.

19. R. Vischer, op. cit., p. 79.

20. A. Bazin, Jean Renoir, Champ Libre, Paris 1971, pp. 46-7.

21. Cf. Jean-Honoré Fragonard Les hasards heureux de l'escarpolette ou La Balançoire (huile sur toile, $81 \times 64.2 \mathrm{~cm}, 1767$, Wallace Collection, Londres), et Auguste Renoir La Balançoire (huile sur toile, $92 \mathrm{~cm} \mathrm{x} 73 \mathrm{~cm}, 1876$, Paris, Musée d'Orsay). Pour une introduction générale, et notamment sur le thème du Faune chez Maupassant, Olivier Curchod, Partie de campagne. Etude critique, Nathan, Paris 1995.

22. A. Bazin, op. cit. pp. 234-6. La notule est de Jacques Domiol-Valcroze.

23. Par là, on serait amené à une lecture Gender Studies, ou politique: E. Manning, Politics of Touch : Sense, Movement, Sovereignty, Minnesota UP, Minnesota 2007.

24. R. Vischer, op. cit., p. 74-5. Cf. M. Merleau-Ponty, L'oil et l'esprit, Gallimard, Paris 1964, p. 23.

25. R. Vischer, op. cit., p. 76. Sur la réversibilité esthésique du « sujet cinétique » au cinéma, V. Sobchack, Carnal Thoughts, cit., p.78-9

26. P. Valéry, « Philosophie de la danse » (1936), đuvres complètes, vol. I, éd. J. Hytier, Gallimard, Paris 1957, p. 1398.

27. Met. IV, 1011a, Probl. XXXI, 11, $958 \mathrm{~b}$ ss, de Somniis 460 b 20-23

28. H. Plessner, « Über die Verkörperungsfunktion der Sinne » (1953), in Gesammelte Schriften, Bd. III, hrsg. von G. Dux, O. Marquard, E. Ströker unter mitwirkung von R. W. Schmidt, A. Wetterer, M.-J. Zemlin, Suhrkamp, Frankfurt a. M. 1980, p. 370-383. Parmi les nombreux renvois au philosophe 
allemand, V. Gallese, "The two sides of Mimesis. Girard's mimetic theory, embodied simulation and social identification ", Journal of Consciousness Studies, vol. 16, n. 4, April 2009, p. 5-6.

29. Au Premier Mot liquide, 1923, huile sur plâtre puis trasféré sur toile, $232 \times 167 \mathrm{~cm}$, Dusseldorf, Kunstsammlung, Nordhein-Westfalen.

30. Ch. Strokes, «The scientific Methods of Max Ernst : His Use of the scientific Subjects from La Nature », The Art Bullettin, vol. 62, no. 3, September 1980, p. 453-465.

31. R. Vischer, op. cit., p. 77 .

32. Ibid.

33. E. Panofsky, Contribution au problème de la description d'oeuvres appartenant aux arts plastiques (1932), in La perspective comme forme symbolique, éd. fr. par G. Ballangé, Minuit, Paris 1975, p. 236-7.

34. P. Valéry, Berthe Morisot (1926), Euvres complètes, vol. II, éd. J. Hytier, Gallimard, Paris 1960, p. 1303.

35. $\mathrm{Si}$, chez Aristote, il suffirait cligner deux fois l'œil pour dissoudre l'illusion tactile et rétablir un bon usage de la vue et du toucher, Ernst dramatise au contraire l'effet pseudo-cinétique de répétition de l'image, télescopant les techniques du montage et les technologies stéréoscopiques des dispositifs visuels, tantôt scientifiques, tantôt populaires et érotiques. Sur cela, j'ose renvoyer à Filippo Fimiani, Fantasmi dell'arte, Liguori, Naples 2012, p. 72 sq.

36. Cf. W. Spies, Max Ernst, 1950-1970 : The Return of La Belle Jardinière, Harry N. Abrams, New York 1971, p. 48, 53 ; E. Legge, Max Ernst : Psychoanalytic Sources, UMI Research Press, Ann Arbor1989, pp. 106-107, 111-113 ; pour Freud, Le délire et les rêves dans la Gradiva de W. Jensen (1907), tr. fr. par M. Bonaparte, préf. J. Pontalis, Gallimard, Paris 1993, p. 34.

37. Cf. G. Didi-Huberman, L'image survivante, Minuit, Paris 2002, pp. 257-9; sur la Nymphe warburghienne et le roman de Nabokov, publié en 1955 et située entre 1947 et 1952, dont Kubrik tirera le long-métrage célèbre en 1962, R. Calasso, La follia che viene dalle Ninfe, Adelphi, Milano 2005, pp. 45-50, et A. Sbrilli, « Le mani fiorentine di Lolita. Coincidenze warburghiane in Nabokov (e viceversa) », Engramma, n. 43, 2005. URL : http://.engramma.it/ engramma_v4/rivista/saggio/43/043_sbrilli_nabokov.html.

38. Sur la ikonische Differenz en tant qu'a priori de toute ontologie, phénoménologie et iconologie, de l'image, je ne peux que renvoyer aux travaux de Gottfried Boehm, notamment Wie Bilder Sinn erzeugen. Die Macht des Zeigens, Berlin University Press, Berlin 2007, et, pour une dernière synthèse, « Ikonische Differenz », Rheinsprung 11. Zeitschrift für Bildkritik, n. 1, 2011, pp. 170-6. URL : http://rheinsprung11.unibas.ch/archiv/ausgabe-01/glossar/ ikonische-differenz.html

39. R. Arnheim, Art and visual Perception. Psychology of creative Eye, cit., pp. 53,57 ss., 63.

40. M. Merleau-Ponty, Phénoménologie de la Perception, Paris, Gallimard 1945, pp. 335-6.

41. Deleuze, d'après Wölfflin, souligne l'interaction entre ante-plan et retro-plan justement chez Renoir et Une partie de campagne : G. Deleuze, Image-mouvement I, op.cit., p. 42-3, note 25.

42. S. Mallarmé, « Ballets », cit., p. 306.

43. M. Merleau-Ponty, Phénoménologie de la Perception, cit., p. 325.

44. S. Mallarmé, « Ballets », cit., p. 303.

45. M. Merleau-Ponty, Phénoménologie de la Perception, cit., p. 337 sq.

46. J. Barker, Tactile Eye, cit., p. 79.

47. L'expression est de Paul Claudel, « Njinski » (1927), Cuuvres en Prose, éd. J. Petit et Ch. Galpérine, Gallimard, Paris 1960, p. 386.

48. Cf. L.U. Marks, The Skin of the Film : Intercultural Cinema, Embodiment, and the Senses, Duke University Press, London 1999, pp. 162 sq., A. Lant, « Haptical Cinema », October, vol. 74 n. 102, Autumn 1995, p. 45-73; G. Deleuze, Cinéma II. L'Image-temps, Minuit, Paris 1985, p. 167-8.

49. R. Arnheim, « Melancholy Unshaped » (1963), in Toward a Psychology of Art, University of California Press, Berkeley 1966, pp. 189-190. Cf. N. Baumbach, « Nature Caught in the Act : On the Transformation of an Idea of Art in Early Cinema », Comparative Critical Studies, vol. 6, no. 3, October 2009, pp. 373-383 ; sur le vent parmi les feuilles comme « scène primitive » de la photographie et de la préhistoire du cinéma, S. Kracauer, Theory of Film : The Redemption of Physical Reality, Princeton University Press, Princeton 1960, pp. 47, 74-5, 85-7, 117, et déjà G. Sadoul, Histoire générale du cinéma. Tome 1. L'invention du cinéma, Denoël, Paris 1946, pp. 49 sq. Cf. aussi Ch. Keathley, Cinephilia and History, or The Wind in the Trees, Indiana University Press, Bloomington 2006, pp. 29-53, et J. Barker, Tactile Eye, cit., p. 149-152.

50. J'ai approfondi cela ailleurs : cf. F. Fimiani, Fantasmi dell'arte, cit., pp. 77 sq.

51. J-P. Sartre, La nausée (1938), Euvres Romanesques, éd. M. Contat et M. Rybalka, Gallimard, Paris 1981, p. 156. 
INDEX

Mots-clés : Mad Men, empathie d'activité, fiction, Einfühlung, figura serpentinata, illusion, images en mouvement

\section{AUTEUR}

\section{FILIPPO FIMIANI}

Professeur associé d'Esthétique à l'Université de Salerne, il est membre de la Società Italiana di Estetica (SIE), d'Æsthetica. Art et Philosophie (AES), de l'Institut Arts, Créations, Théories, Esthétique (ACTE) de l'Université Paris 1 Panthéon-Sorbonne-CNRS, et du Centre de Recherches sur les Arts et le Langage CRAL) de l'EHESS du Laboratoire L'Antique, le Moderne (LAMO) du Centre de recherche Textes, Langages, Imaginaires/ Marges Modernités Antiquités (TLI/MMA) de l'Université de Nantes, de l'Osservatorio Italiano su Estetica e Storia dell'Arte et de l'InterUniversity Research Center for Social and Communication Network Analysis and Knowledge Communication. Il est l'auteur notamment de Fantasmi dell'arte. Sei storie con spettatore. Napoli 2012, Forme informi. Studi di poetiche del visuale, Genova 200, Poetica Mundi. Estetica e ontologia delle forme in Paul Claudel, Palermo 2001, Poetiche e genealogie. Claudel, Valéry, Nietzsche, Napoli 2000, La sovranità dell'evento. Saggio su Charles Péguy, Milan 1994. Pour sa bibliographie complète voir : http://dsc.unisa.it/fimiani/Fr 